\title{
Progress on a Study of Target-Horn System for Muon Colliders
}

\section{Nicolette Muldrow*, Pavel Snopok, IIT; Katsuya Yonehara, Fermilab}

\section{Motivation}

The goal is to make a multi-MW Target System. The first challenges in making this system are the heat and radiation treatment and the phase space manipulation for cooling channels. For the heat and radiation treatment for NuMl, 1/3 of total beam power is deposited in Target hall, 1/3 in Decay Pipe, and 1/3 in Hadron Absorber. Also, for NuMl, graphite target was activated $75 \mathrm{Rad} / \mathrm{hr}$ on contact after servicing 2 yrs with $10^{21}$ POT. For the phase space manipulation for cooling channels achromatic pion capture optics is required. We are Investigating horn-based systems. The sub-MW target system exists and can be utilized. Our first steps are to make a conceptual model and run numerical simulation to validate a new horn concept.

\section{Simulation Results}
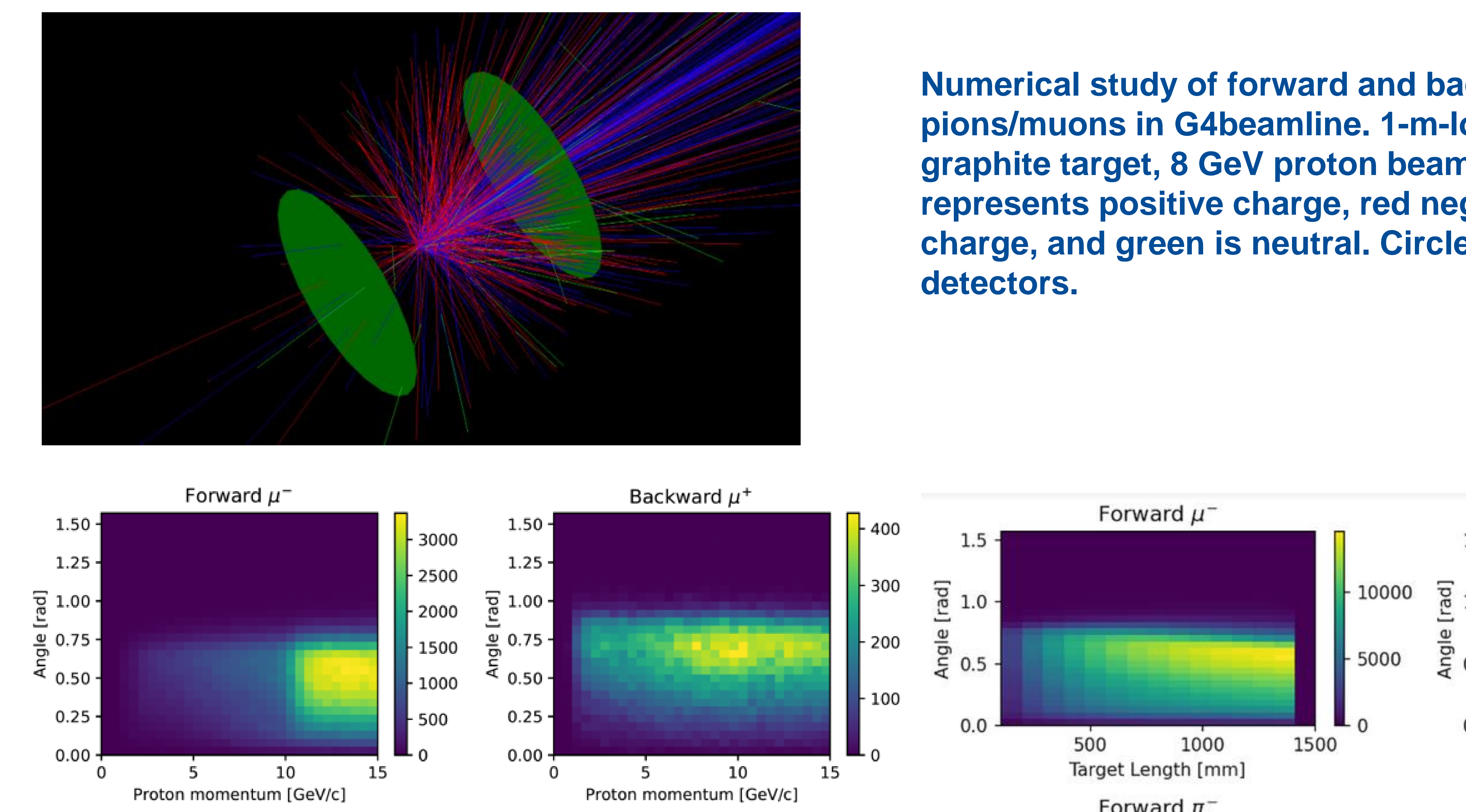

Numerical study of forward and backward pions/muons in G4beamline. 1-m-long represents posit, $8 \mathrm{GeV}$ proton beam. Blue charge, and green is neutral. Circles are virtual detectors.

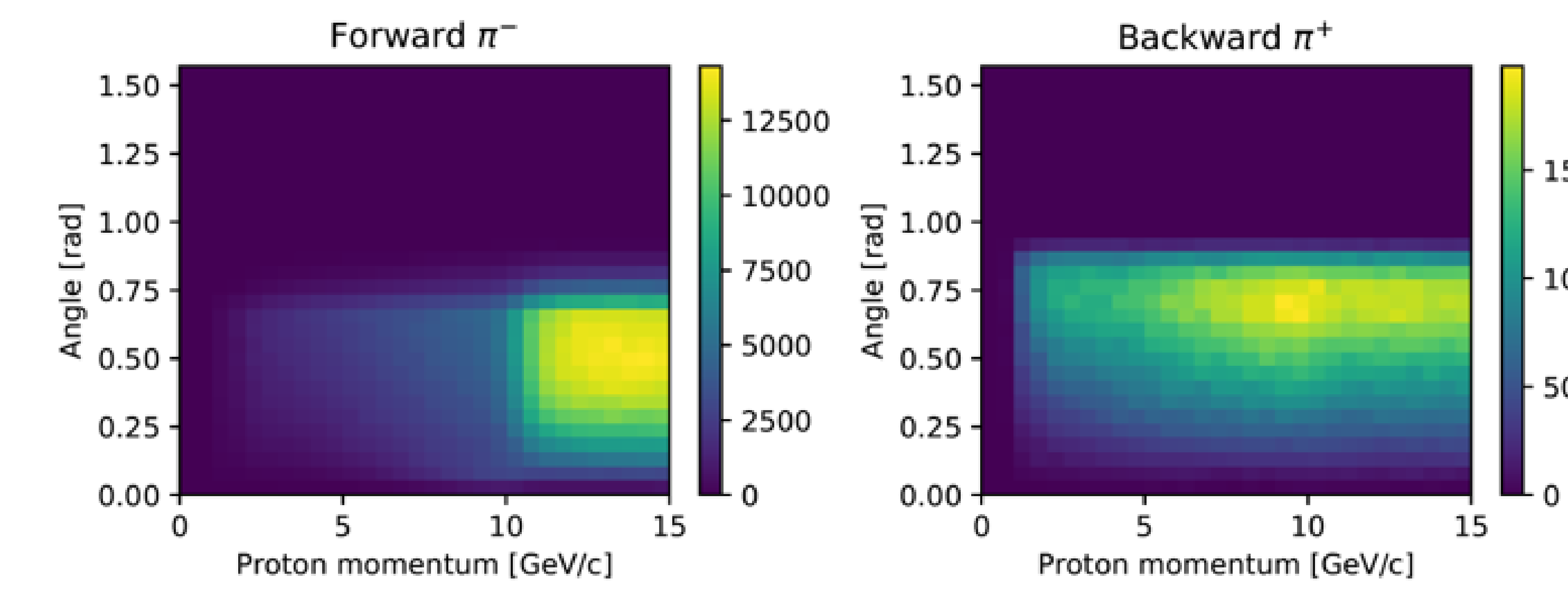

Preliminary results plotting angle vs proton momentum 2. Momentum cut $100 \leq p \leq 400 \mathrm{MeV} / \mathrm{c}$

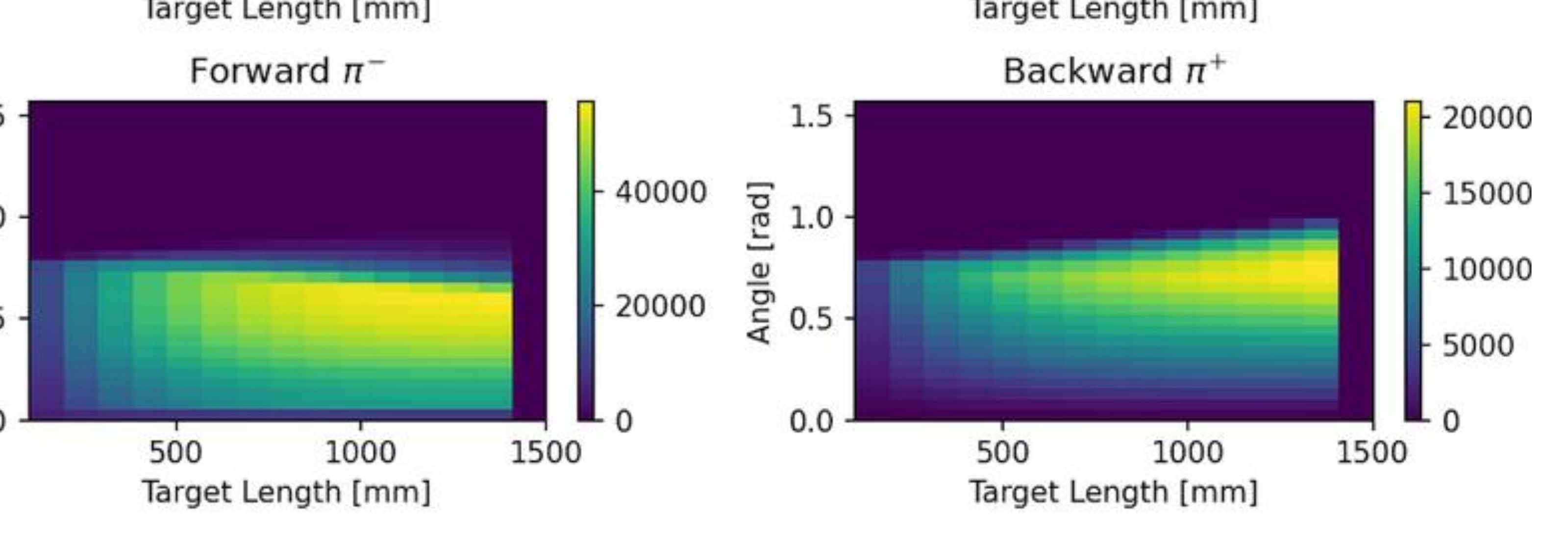

Preliminary results for angle vs target length, $100<\mathrm{d}<=1500 \mathrm{~mm}$.

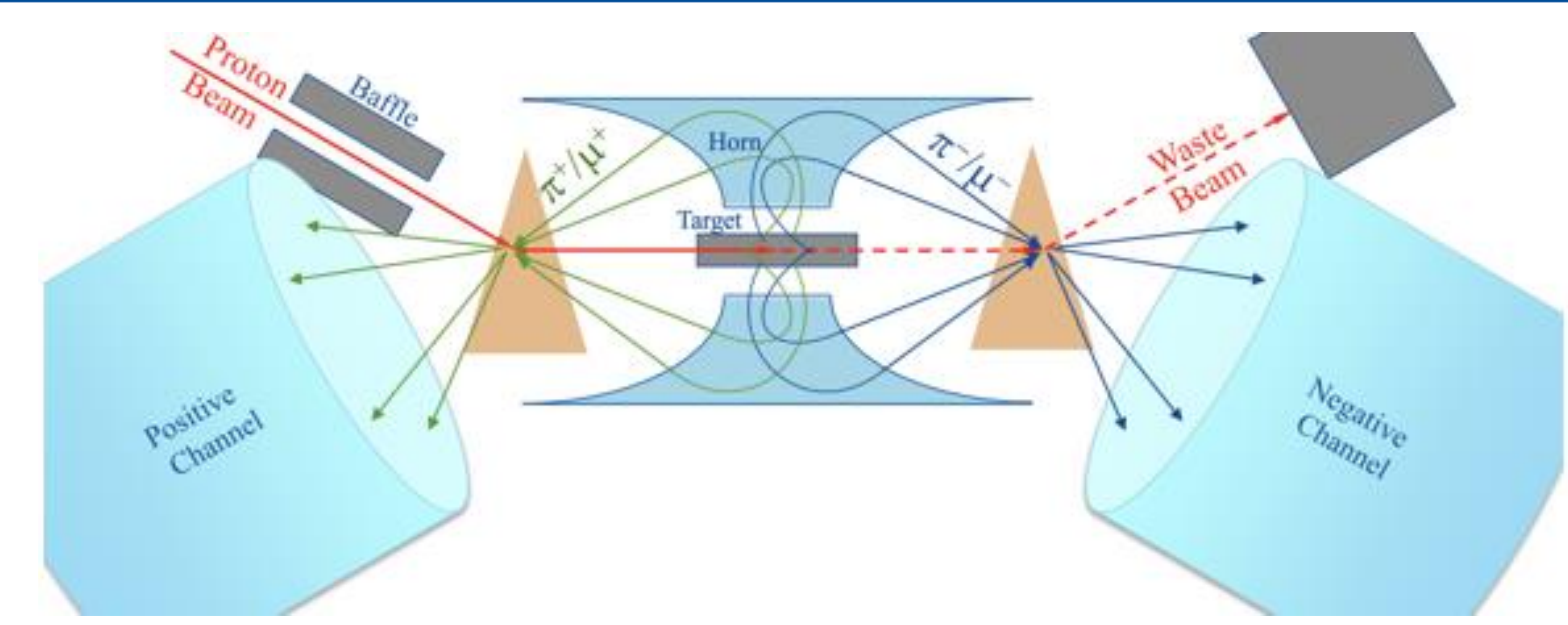

Horn Capture of Positives and Negatives

Direct proton beam onto the target surrounded by a symmetric horn. Focus negative/positive secondaries forward/backward. Dipoles separate incident proton beam from backward positive beam and waste proton beam from forward negative beam. Direct beams into cooling channels. (Not to scale)

Analytical Model

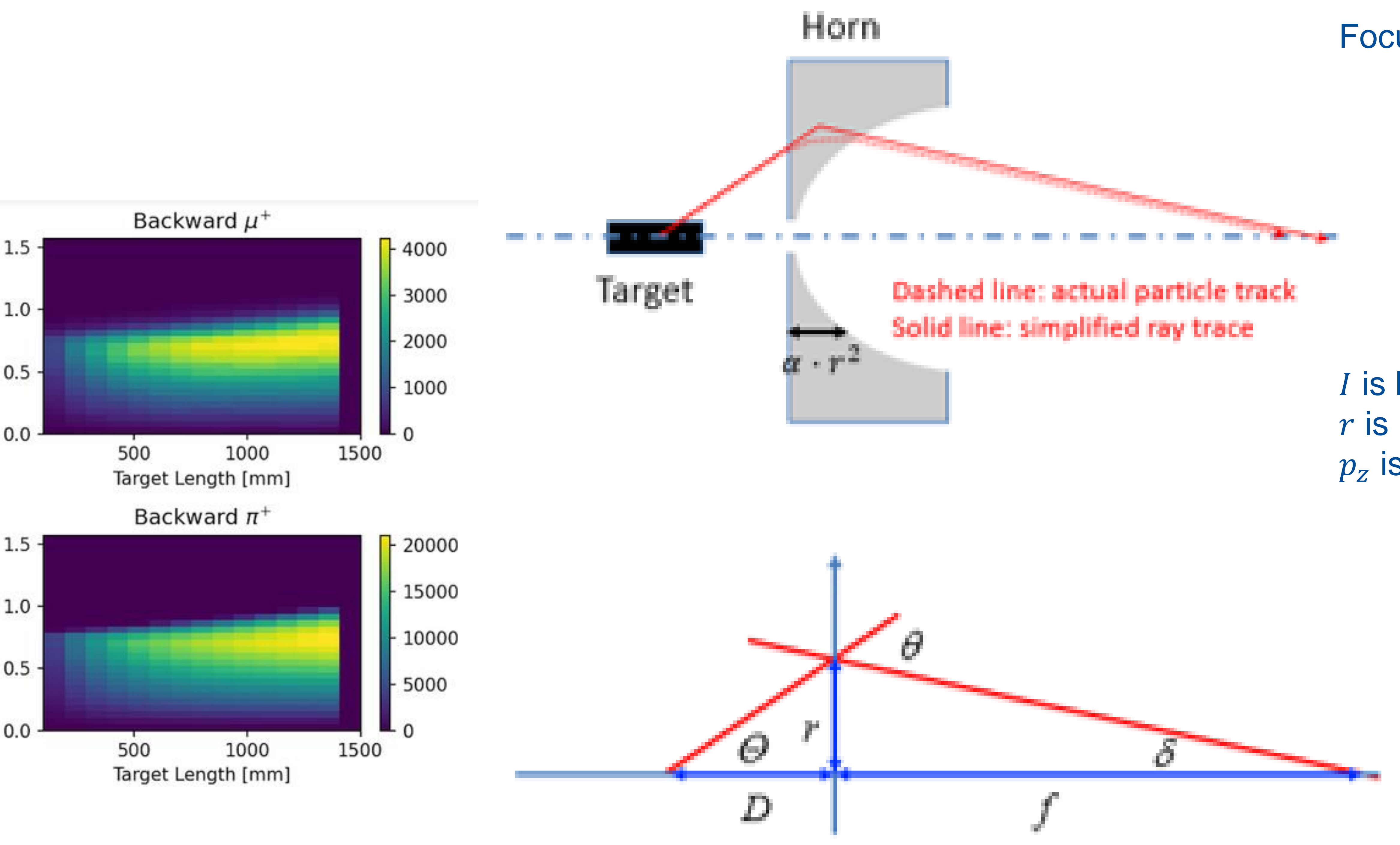

Focusing Force

Toy Model

- Horn focusing force $\theta=\frac{e b_{\varphi} l}{p_{z}} \propto \frac{I \cdot r}{p_{z}}$

- Cross-sectional shape of horn $z=\alpha \cdot r^{2}$

- Focusing geometry can be fixed from known dimensions with a simplified model 